\title{
Solvent Environment Revealed by Positively Chirped Pulses
}

\author{
Arkaprabha Konar, ${ }^{\dagger}$ Vadim V. Lozovoy, ${ }^{\dagger}$ and Marcos Dantus ${ }^{* \dagger, \dagger}$ \\ ${ }^{\dagger}$ Department of Chemistry and ${ }^{\star}$ Department of Physics and Astronomy, Michigan State University, East Lansing, Michigan 48824, \\ United States
}

\section{Supporting Information}

\begin{abstract}
The spectroscopy of large organic molecules and biomolecules in solution has been investigated using various time-resolved and frequency-resolved techniques. Of particular interest is the early response of the molecule and the solvent, which is difficult to study due to the ambiguity in assigning and differentiating inter- and intramolecular contributions to the electronic and vibrational populations and coherence. Our measurements compare the yield of fluorescence and stimulated emission for two laser dyes IR144 and IR125 as a function of chirp. While negatively chirped pulses are insensitive to solvent viscosity, positively chirped pulses are found to be uniquely sensitive probes of solvent viscosity. The fluorescence maximum for IR125 is observed near transform-limited pulses; however, for IR144, it is observed for positively chirped pulses once the pulses have been stretched to hundreds of femtoseconds. We conclude that chirped pulse spectroscopy is a simple one-beam method that is sensitive to early solvation dynamics.
\end{abstract}

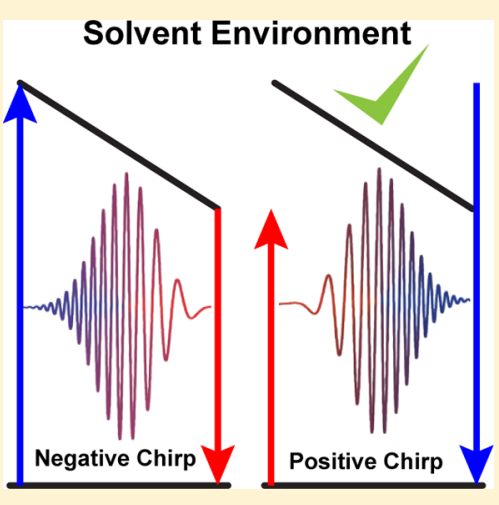

SECTION: Spectroscopy, Photochemistry, and Excited States

$\mathrm{U}$ nderstanding molecular dynamics soon after photon absorption, taking into account the solvent environment surrounding the molecule, is central to predicting the course of chemical reactions and biophysical processes. The relevant time scales regarding the coherent response to photoexcitation in solution are determined by the inter- and intramolecular interactions and their corresponding energy fluctuations. ${ }^{1-11}$ The multiple processes occurring during this time, convoluted by inhomogeneous broadening as well as the spectral and temporal response function of the experimental setup, complicate assignment of the observed decay processes. Here, we focus on the optical response from solvated IR125 and IR144, which have been studied in solution as a function of temperature and solvent. The organic dye molecule IR125 undergoes nonpolar solvation, while IR144 undergoes polar solvation given the reduction in its dipole moment upon excitation. 8 The difference between the molecules is attributed to the piperazine functional group in IR144. Three-pulse photon-echo peak shift (3PEPS) measurements revealed a solvent independent coherent response for times $<0.1 \mathrm{ps}$, described as inertial, followed by a solvent dependent component changing from 4.04 ps to 1.36 ps in ethylene glycol at $297 \mathrm{~K}$ and $397 \mathrm{~K}^{13}$ Fluorescence upconversion measurements with broad-band detection also can yield insights into the intra- and intermolecular dynamics that are comparable to those for other nonlinear optical techniques. ${ }^{14,15}$ Our goal is to find spectroscopic probes of the solvation environment that are sensitive and easier to implement in a microscope; in particular, we evaluate here chirped femtosecond pulses. These singlebeam methods will be of paramount importance when investigating microenvironment effects on single molecules due to the relative ease of the experimental implementation.
The asymmetric response ${ }^{7}$ of fluorescence to chirped femtosecond pulses was first observed by Shank and coworkers. ${ }^{16}$ Fluorescence depletion was observed for negatively chirped pulses and explained as an intrapulse pump-dump process that follows the nascent wave packet in the upper excited state as the carrier frequency shifts to lower energies. That process is not available for positively chirped pulses, for which the carrier frequency shifts to higher energies. The depletion mechanism has been used for controlling population transfer in multilevel systems owing to its robustness with respect to fluctuations. ${ }^{17-19}$

Different qualitative and quantitative interpretations of the chirp dependence of molecular fluorescence have been advanced; Cao et al. $^{20}$ developed an intrapulse three-level model, while Fainberg and co-workers have worked on including non-Markovian relaxation in the existing theory. ${ }^{21-23}$

Our work using chirped pulses established that the changes observed are quadratic, with laser intensity even for excitation probabilities in the range of $0.02-5 \% .{ }^{24}$ All experiments showed enhanced (compared to transform-limited pulses) fluorescence with positively chirped pulses and enhanced stimulated emission (compared to transform-limited) with negatively chirped pulses. ${ }^{24}$ The main difference between stimulated emission and fluorescence is that stimulated emission is a coherent $\chi^{(3)}$ process that gives rise to a thirdorder polarization. The emission is greatest when the initial excitation has not undergone intramolecular vibrational randomization (IVR) and dephasing. This however is not

Received: February 11, 2014

Accepted: February 14, 2014 
necessarily true for the pump-probe type of experiments, where the probe pulse interrogates a population excited by the pump pulse in a manner that is not phase-sensitive. Fluorescence is a spontaneous emission process occurring after IVR that depends on the population that the chirped pulse achieves. The characteristic fluorescence depletion observed for negative chirped pulses is a result of enhanced stimulated emission, which is favored for the blue-to-red frequency ordering of negatively chirped pulses.

We developed a four-level system shown in Figure 1 and solved it using the Liouville equation without the assumption of

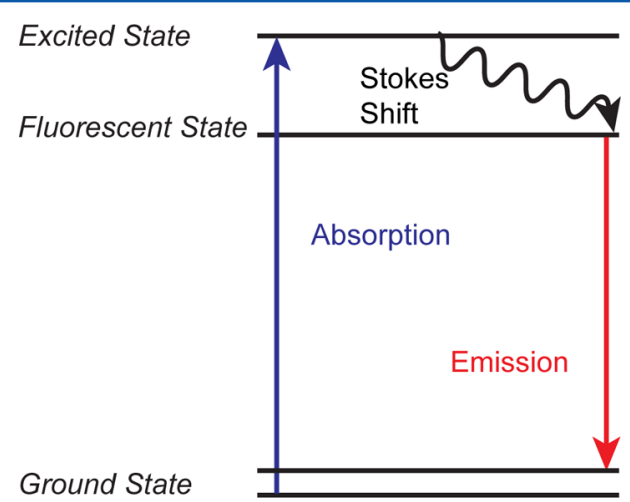

Figure 1. Schematic of the optical processes relevant to chirp dependence measurements. This is the minimum number of states that we found to be required to model our results. There is a difference between coherent stimulated emission, which occurs at higher frequencies, and fluorescence, which occurs spontaneously from a relaxed fluorescent state. weak field interactions, and the theory fit our data well. ${ }^{24} \mathrm{We}$ found that a relaxation rate in the range of 20-30 fs (solution of IR144 in methanol at room temperature) reproduced our data closely.

Measurements of the dependence of the integrated fluorescence intensity (detected at $90^{\circ}$ ) and stimulated emission (detected in the forward direction along the excitation beam) were recorded as a function of chirp. Results for both dyes dissolved in ethylene glycol at different temperatures are shown in Figure 2. A secondary axis denoting the pulse duration of the chirped pulses has been provided in the figures to help elucidate the timing of inter and intramolecular processes taking place during the chirp scans. The data are normalized on the asymptotic values of the chirp effect. For fluorescence (left column), the curves were normalized on the asymptotic values attained for negative chirp, while the stimulated emission curves (right column) were normalized on the positive chirp asymptotes. An alternate method of normalization, where all curves are normalized to match the zero-chirp value, is shown in Figure S1 (Supporting Information).

The changes in stimulated emission and fluorescence as a function of solvent and temperature are shown in Figure $2 a-d$. Measurements for the two different dyes dissolved in ethylene glycol were performed at 278,294 , and $323 \mathrm{~K}$ and have been color coded as blue, black, and red, respectively. The viscosities of ethylene glycol at 5, 20, and $50{ }^{\circ} \mathrm{C}$ are $45.4,19.3$, and 6.55 $\mathrm{mPa}$ s, respectively. ${ }^{25}$ Most importantly, our findings reveal that negative chirp, where historically most of the scientific research has focused, ${ }^{16,17,19,20,26-48}$ is insensitive to solvent viscosity. Negative chirp experiments can be thought of as being similar to pump-probe measurements, in which the bluer wavelength

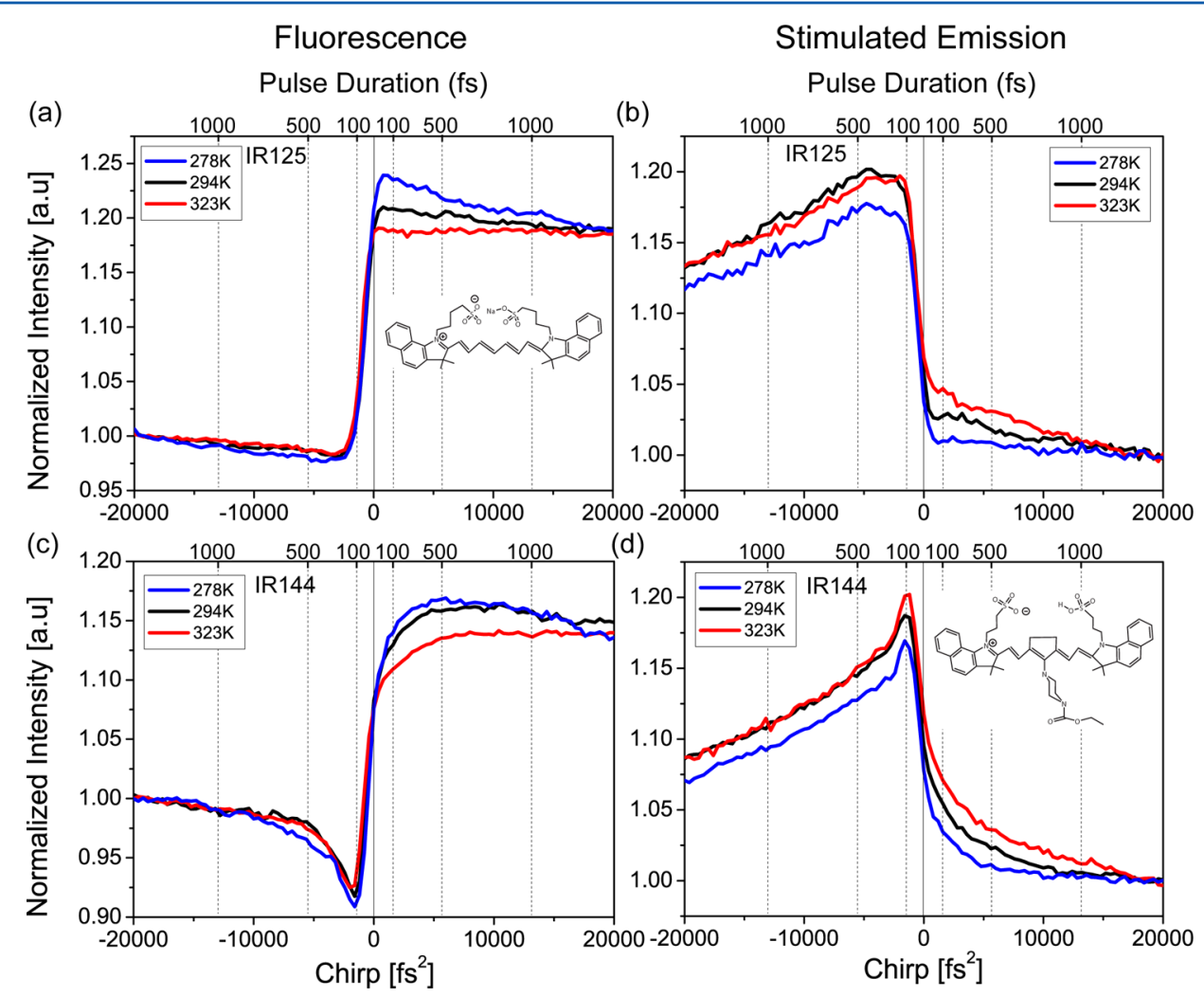

Figure 2. Fluorescence and stimulated emission response to chirped pulses for IR125 (a,b) and for IR144 (c,d), respectively, in ethylene glycol at three temperatures, 278 (blue), 294 (black), and $323 \mathrm{~K}$ (red). The plots have been normalized on the asymptotic value of the chirp effect. 
pump precedes the redder wavelength probe. Negative chirp leads to enhanced stimulated emission and hence lower fluorescence intensity. It is worth noting that the feature found at $-2500 \mathrm{fs}^{2} \sim 200 \mathrm{fs}$ for IR144 in fluorescence and in stimulated emission coincides and is likely the same as a vibrational feature found in the 3PEPS results on IR144 in all solvents at $\mathrm{T}=0.2$ ps. ${ }^{13}$ Positive chirp, because of the time ordering of the frequencies, is not effective for stimulated emission. Interestingly, once the excited state is reached, the time ordering of the frequencies of positively chirped pulses is such that it can stimulate a transition back to the ground state and again back to the excited state.

Positive chirp, on the other hand, yields different dynamics as a function of temperature. When exploring IR125 fluorescence, we find that colder solvent leads to enhanced fluorescence near $800 \mathrm{fs}^{2}$. This enhancement is not observed at higher temperatures. A similar fluorescence enhancement for colder solvent is observed for IR144; however, the maximum fluorescence is reached around $5000 \mathrm{fs}^{2}$, which corresponds to $\sim 500 \mathrm{fs}$. The trends observed in fluorescence are reflected in stimulated emission. The stimulated emission intensity drops sharply for positively chirped pulses for IR125, while the rate is much slower for IR144. This decay rate decreases with increasing temperatures for both molecules. It is important to note that the chirp values found depend on the characteristics of the transform-limited pulses, in particular, the bandwidth.

Our results clearly show sensitivity to the solvent viscosity. The overall difference in the shape and decay rates between the two dyes can be attributed to molecular properties of the probe molecules. IR144 undergoes a change in dipole moment upon excitation and therefore undergoes polar solvation, which depends on solvent reorientation. This accounts for the slower dynamics of IR144 in ethylene glycol. IR125 on the other hand undergoes nonpolar solvation due to the absence of any significant change in dipole moment upon excitation.

The viscoelastic model for nonpolar solvation predicts a rapid viscosity-independent "ballistic" or coherent phononinduced response and slower viscosity-dependent diffusive dynamics that relieve stress following excitation. ${ }^{49}$ The diffusive dynamics have been shown to be sensitive to the vibrational dephasing in viscous media. ${ }^{50}$ Building on the viscoelastic theory, photon-echo studies have tried to distinguish between the fast modulation, spectral diffusion, and quasi-static dynamics based on the scaling of the echo decay time as a function of viscosity. ${ }^{51}$ This distinction is difficult based on the relative insensitivity of the photon-echo measurements. When 3PEPS measurements were fitted to five time constants, no change was observed in the first two fastest time constants (times less than $100 \mathrm{fs}$ ), but a $0.5 \mathrm{fs}$ difference in the peak shift found for picosecond times was reflected in the third and fourth time constants, where the decay rates measured for methanol were found to be three times faster than those involving ethylene glycol. ${ }^{52}$ When ethylene glycol was heated to $397 \mathrm{~K}$, the results found from 3PEPS more closely matched methanol; this is something that is reproduced by our measurements (Figure S2, Supporting Information). Our positive chirp findings for IR125 are consistent with the rapid viscosityindependent coherent phonon response (rise close to zero chirp) followed by the slower viscosity-dependent diffusive dynamics observed as the pulse duration increases. In contrast, the dipolar response of IR144 depends on solvent reorientation, a process that is viscosity-dependent, and thus delays the point where maximum fluorescence is observed.
Shaped pulses, for example chirp as presented in this Letter, are found to be particularly sensitive to the solvent environment, especially during the first few hundred femtoseconds, where previous nonlinear spectroscopic approaches have been less sensitive. We are in the process of simulating these findings in order to place them in the context of modern theoretical methods that take into account the evolution of the density matrix in the presence of a realistic solvation environment. The ability of phase-shaped laser pulses to probe the solvent environment is particularly exciting given the relative ease of these experiments compared to the much more complicated four-wave mixing setups. We plan to take advantage of chirped pulses to probe solvent environment effects of probe molecules in interesting environments such as protein pockets and membranes and under single-molecule conditions.

\section{EXPERIMENTAL SECTION}

The experimental apparatus used for these experiments has been described elsewhere. ${ }^{24}$ Briefly, pulses from a femtosecond regeneratively amplified Ti:Sapphire laser producing $26 \mathrm{~nm}$ fwhm (corresponding to $36 \mathrm{fs}$ when transform-limited) were used. Pulses were compressed and shaped using a pulse shaper (MIIPS-HD, Biophotonic Solutions Inc.) placed after the amplifier. $^{53,54}$ A chirped pulse scan consisted of recording molecular emissions as a function of $\varphi^{\prime \prime}$, the spectral chirp from negative to positive $20000 \mathrm{fs}^{2}$, for $\varphi(\omega)=0.5 \varphi^{\prime \prime}\left(\omega-\omega_{0}\right)^{2}$. The chirp was scanned back and forth to eliminate any systematic errors. Given the initial pulse duration for transformlimited pulses of $36 \mathrm{fs}$, the pulses were stretched to have a maximum pulse duration of $2.17 \mathrm{ps}$ fwhm, according to $\left(\tau / \tau_{\mathrm{TL}}\right)$ $=\left[1+\left(4 \ln 2\left(\varphi^{\prime \prime} / \tau_{\mathrm{TL}}^{2}\right)\right)\right]^{1 / 2}$. In a sense, a chirp scan can be interpreted as two-color time-resolved measurements for which early changes such as those occurring at $100 \mathrm{fs}$ are observed near $1000 \mathrm{fs}^{2}$. For negative chirps, high frequencies arrive before low frequencies, and for positive chirps, the order is reversed. For all experiments, unfocused laser pulses centered at $800 \mathrm{~nm}$ were used. The attenuated and shaped amplified pulses were energetic enough to achieve peak intensities (when transform-limited) of $4 \times 10^{9} \mathrm{~W} / \mathrm{cm}^{2}$. The relatively low repetition rate of the laser $(1 \mathrm{kHz}$ compared to oscillators with $100 \mathrm{MHz}$ rates) permits sufficient time for relaxation between pulses and greatly reduces the need for flow in our measurements. The solutions with optical density of $<0.3$ were placed in $2 \mathrm{~mm}$ cuvettes in order to minimize phase distortion and reabsorption effects. The cuvette was placed in a metal sample holder connected to an aluminum base. For the high-temperature measurements, the base was heated, and the temperature was allowed to equilibrate. For achieving the low temperatures, the whole assembly was immersed in an ice bath, and a constant flow of dry $\mathrm{N}_{2}$ was used to prevent condensation on the cuvette.

The results presented here depend on the ability to deliver pulses that are free of high-order dispersion, the long-term stability of the laser output, and finding the concentration and laser intensity that reduced possible local heating effects to a level that is below our measurement ability. The reproducibility of our data was tested by repeating all of the measurements on different days and comparing them without any normalization. The reader can see the results when normalized to the fluorescence or stimulated emission detected for transformlimited pulses (see Figure S1, Supporting Information) to confirm that all curves for negative chirp are parallel (no sensitivity to the solvent) while the curves obtained for positive 
chirp are found to depend on solvent viscosity. We also include in the Supporting Information results obtained for methanol and compare them with results obtained for ethylene glycol at $323 \mathrm{~K}$ (see Figure S2). We find excellent agreement for IR125 and IR144, although IR144 does show a faster loss of stimulated emission for methanol. Finally, given that the results presented depend on linear emission, we checked for effects of local heating by acquiring data at laser repetition rates of $1 \mathrm{kHz}$ and $100 \mathrm{~Hz}$ while looking for deviations in the observed chirp dependence. We found that for high concentration and high laser intensity, some deviations were noted. However, when the optical density was reduced to $\angle 0.3 \mathrm{OD}$ and the pulse energy was reduced to $40 \mu \mathrm{J}(1 \mathrm{kHz})$, deviations were no longer observed (Figure S3, Supporting Information). On a technical note, changing the repetition rate of our laser caused a change in the output phase characteristics, and we had to recompensate in order to get transform-limited pulses before repeating the measurements.

\section{ASSOCIATED CONTENT}

\section{S Supporting Information}

Figure S1 shows our measurements normalized such that all signals match at zero chirp, to show that the method used to normalize does not change the observed trends. Figure S2 compares results for ethylene glycol at $323 \mathrm{~K}$ and methanol, which for IR125 are found to be nearly identical and for IR144 they are found to be very similar. Figure S3 shows results obtained at laser repetition rates of $1 \mathrm{kHz}$ and $100 \mathrm{~Hz}$. Both are found identical, and this indicates laser heating effects in our measurements are negligible. This material is available free of charge via the Internet at http://pubs.acs.org.

\section{AUTHOR INFORMATION}

\section{Corresponding Author}

*E-mail: dantus@msu.edu.

\section{Notes}

The authors declare no competing financial interest.

\section{ACKNOWLEDGMENTS}

We thank DOE SISGR (DE-SC0002325) and Dr. Jeff Krause (Program Manager) for supporting this research. We would also like to thank Prof. Warren F. Beck for lending us the sample cell holder and for valuable discussions regarding solvation phenomena.

\section{REFERENCES}

(1) Mukamel, S., Principles of Nonlinear Optical Spectroscopy; Oxford University Press: New York, 1995.

(2) Joo, T.; Albrecht, A. C. Electronic Dephasing Studies of Molecules in Solution at Room-Temperature by Femtosecond Degenerate 4-Wave-Mixing. Chem. Phys. 1993, 176, 233-247.

(3) Jimenez, R.; Fleming, G. R; Kumar, P. V.; Maroncelli, M. Femtosecond Solvation Dynamics of Water. Nature 1994, 369, 471473.

(4) deBoeij, W. P.; Pshenichnikov, M. S.; Wiersma, D. A. SystemBath Correlation Function Probed by Conventional and Time-Gated Stimulated Photon Echo. J. Phys. Chem. 1996, 100, 11806-11823.

(5) de Boeij, W. P.; Pshenichnikov, M. S.; Wiersma, D. A. Ultrafast Solvation Dynamics Explored by Femtosecond Photon Echo Spectroscopies. Annu. Rev. Phys. Chem. 1998, 49, 99-123.

(6) Xu, Q. H.; Scholes, G. D.; Yang, M.; Fleming, G. R. Probing Solvation and Reaction Coordinates of Ultrafast Photoinduced Electron-Transfer Reactions Using Nonlinear Spectroscopies: Rhod- amine 6G in Electron-Donating Solvents. J. Phys. Chem. A 1999, 103, $10348-10358$.

(7) Gunaratne, T. C.; Zhu, X.; Lozovoy, V. V.; Dantus, M. Symmetry of Nonlinear Optical Response to Time Inversion of Shaped Femtosecond Pulses as a Clock of Ultrafast Dynamics. Chem. Phys. 2007, 338, 259-267.

(8) Yu, A. C.; Tolbert, C. A.; Farrow, D. A.; Jonas, D. M. Solvatochromism and Solvation Dynamics of Structurally Related Cyanine Dyes. J. Phys. Chem. A 2002, 106, 9407-9419.

(9) Jonas, D. M. Two-Dimensional Femtosecond Spectroscopy. Annu. Rev. Phys. Chem. 2003, 54, 425-463.

(10) Schlau-Cohen, G. S.; Ishizaki, A.; Calhoun, T. R.; Ginsberg, N. S.; Ballottari, M.; Bassi, R.; Fleming, G. R. Elucidation of the Timescales and Origins of Quantum Electronic Coherence in LHCII. Nat. Chem. 2012, 4, 389-395.

(11) Collini, E.; Wong, C. Y.; Wilk, K. E.; Curmi, P. M. G.; Brumer, P.; Scholes, G. D. Coherently Wired Light-Harvesting in Photosynthetic Marine Algae at Ambient Temperature. Nature 2010, 463, 644-U69.

(12) Bertolino, C. A.; Ferrari, A. M.; Barolo, C.; Viscardi, G.; Caputo, G.; Coluccia, S. Solvent Effect on Indocyanine Dyes: A Computational Approach. Chem. Phys. 2006, 330, 52-59.

(13) Passino, S. A.; Nagasawa, Y.; Joo, T.; Fleming, G. R. Three-Pulse Echo Peak Shift Studies of Polar Solvation Dynamics. J. Phys. Chem. A 1997, 101, 725-731.

(14) Ajdarzadeh, A.; Consani, C.; Bräm, O.; Tortschanoff, A.; Cannizzo, A.; Chergui, M. Ultraviolet Transient Absorption, Transient Grating and Photon Echo Studies of Aqueous Tryptophan. Chem. Phys. 2013, 422, 47-52.

(15) Braem, O.; Penfold, T. J.; Cannizzo, A.; Chergui, M.; Femtosecond, A. Fluorescence Study of Vibrational Relaxation and Cooling Dynamics of UV Dyes. Phys. Chem. Chem. Phys. 2012, 14, 3513-3519.

(16) Cerullo, G.; Bardeen, C. J.; Wang, Q.; Shank, C. V. High-power Femtosecond Chirped Pulse Excitation of Molecules in Solution. Chem. Phys. Lett. 1996, 262, 362-368.

(17) Bardeen, C. J.; Yakovlev, V. V.; Wilson, K. R.; Carpenter, S. D.; Weber, P. M.; Warren, W. S. Feedback Quantum Control of Molecular Electronic Population Transfer. Chem. Phys. Lett. 1997, 280, 151-158.

(18) Cao, J. S.; Bardeen, C. J.; Wilson, K. R. Molecular "Pi Pulse" for Total Inversion of Electronic State Population. Phys. Rev. Lett. 1998, $80,1406-1409$.

(19) Bardeen, C. J.; Cao, J. S.; Brown, F. L. H.; Wilson, K. R. Using Time-Dependent Rate Equations to Describe Chirped Pulse Excitation in Condensed Phases. Chem. Phys. Lett. 1999, 302, 405-410.

(20) Cao, J. S.; Che, J. W.; Wilson, K. R. Intrapulse Dynamical Effects in Multiphoton Processes: Theoretical Analysis. J. Phys. Chem. A 1998, 102, 4284-4290.

(21) Fainberg, B. D.; Gorbunov, V. A. Coherent Population Transfer in Molecules Coupled with a Dissipative Environment by an Intense Ultrashort Chirped Pulse. J. Chem. Phys. 2002, 117, 7222-7232.

(22) Fainberg, B. D.; Gorbunov, V. A. Coherent Population Transfer in Molecules Coupled with a Dissipative Environment by Intense Ultrashort Chirped Pulse. II. A Simple Model. J. Chem. Phys. 2004, $121,8748-8754$.

(23) Katz, G.; Ratner, M. A.; Kosloff, R. Control by Decoherence: Weak Field Control of an Excited State Objective. New J. Phys. 2010, $12,015003 / 1-015003 / 13$.

(24) Konar, A.; Lozovoy, V. V.; Dantus, M. Solvation Stokes-Shift Dynamics Studied by Chirped Femtosecond Laser Pulses. J. Phys. Chem. Lett. 2012, 3, 2458-2464.

(25) Haynes, W. M.; Bruno, T. J.; Lide, D. R., CRC Handbook of Chemistry and Physics, 94th ed; CRC Press: Boca Raton, FL, 20132014; pp 6-232.

(26) Nibbering, E. T. J.; Wiersma, D. A.; Duppen, K. Ultrafast Nonlinear Spectroscopy with Chirped Optical Pulses. Phys. Rev. Lett. 1992, 68, 514-517.

(27) Krause, J. L.; Whitnell, R. M.; Wilson, K. R.; Yan, Y. J.; Mukamel, S. Optical Control of Molecular-Dynamics - Molecular 
Cannons, Reflectrons, and Wave-Packet Focusers. J. Chem. Phys. 1993, 99, 6562-6578.

(28) Ashworth, S. H.; Hasche, T.; Woerner, M.; Riedle, E.; Elsaesser, T. Vibronic Excitations of Large Molecules in Solution Studied by Two-Color Pump-Probe Experiments on the 20 fs Time Scale. J. Chem. Phys. 1996, 104, 5761-5769.

(29) Bardeen, C. J.; Yakovlev, V. V.; Squier, J. A.; Wilson, K. R. Quantum Control of Population Transfer in Green Fluorescent Protein by Using Chirped Femtosecond Pulses. J. Am. Chem. Soc. 1998, 120, 13023-13027.

(30) Yakovlev, V. V.; Bardeen, C. J.; Che, J. W.; Cao, J. S.; Wilson, K. R. Chirped Pulse Enhancement of Multiphoton Absorption in Molecular Iodine. J. Chem. Phys. 1998, 108, 2309-2313.

(31) Mishima, K.; Hayashi, M.; Lin, J. T.; Yamashita, K.; Lin, S. H. A Numerical Study on Vibronic and Vibrational Dynamics Generated by Chirped Laser Pulses in the Presence of Relaxation Processes. Chem. Phys. Lett. 1999, 309, 279-286.

(32) Fainberg, B. D.; Narbaev, V. Chirped Pulse Excitation in Condensed Phases Involving Intramolecular Modes Studied by Double-Sided Feynman Diagrams for Fast Optical Dephasing. J. Chem. Phys. 2000, 113, 8113-8124.

(33) Misawa, K.; Kobayashi, T. Wave-Packet Dynamics in a Cyanine Dye Molecule Excited with Femtosecond Chirped Pulses. J. Chem. Phys. 2000, 113, 7546-7553.

(34) Lee, S. H.; Jung, K. H.; Sung, J. H.; Hong, K. H.; Nam, C. H. Adaptive Quantum Control of DCM Fluorescence in the Liquid Phase. J. Chem. Phys. 2002, 117, 9858-9861.

(35) Hashimoto, N. T.; Misawa, K.; Lang, R. Three-Level Picture for Chirp-Dependent Fluorescence Yields under Femtosecond Optical Pulse Irradiation. App. Phys. Lett. 2003, 82, 2749-2751.

(36) Nahmias, O.; Bismuth, O.; Shoshana, O.; Ruhman, S. Tracking Excited State Dynamics with Coherent Control: Automated Limiting of Population Transfer in LDS750. J. Phys. Chem. A 2005, 109, 82468253.

(37) Zhang, S.; Sun, Z. R.; Zhang, X. Y.; Xu, Y.; Wang, Z. G.; Xu, Z. Z.; Li, R. X. Optimal Feedback Control of Two-Photon Fluorescence in Coumarin 515 Based on Genetic Algorithm. Chem. Phys. Lett. 2005, $415,346-350$.

(38) Vogt, G.; Nuernberger, P.; Selle, R.; Dimler, F.; Brixner, T.; Gerber, G. Analysis of Femtosecond Quantum Control Mechanisms with Colored Double Pulses. Phys. Rev. A 2006, 74, 3.

(39) Karasawa, N.; Tada, K.; Ohmori, H. The Comparison Between Experiment and Calculation of the Chirp-Controlled Raman SelfFrequency Shift in a Photonic Crystal Fiber. IEEE Photonics Technol. Lett. 2007, 19, 1292-1294.

(40) Carroll, E. C.; Florean, A. C.; Bucksbaurn, P. H.; Spears, K. G.; Sension, R. J. Phase Control of the Competition Between Electronic Transitions in a Solvated Laser Dye. Chem. Phys. 2008, 350, 75-86.

(41) Lerch, E.-B. W.; Dai, X.; Torres, E. A.; Ballard, J. B.; Stauffer, H. U.; Leone, S. R. Manipulation of Ro-vibronic Wave Packet Composition Using Chirped Ultrafast Laser Pulses. J. Phys. B: At. Mol. Opt. Phys. 2008, 41, 074015/1-074015/7.

(42) Marquetand, P.; Nuernberger, P.; Brixner, T.; Engel, V. Molecular Dump Processes Induced by Chirped Laser Pulses. J. Chem. Phys. 2008, 129, 074303/1-074303/11.

(43) Serrat, C.; Biegert, J. Energy Efficient Method for Two-Photon Population Transfer with near-Resonant Chirped Pulses. Opt. Exp. 2008, 16, 19667-19673.

(44) Nuernberger, P. Differences and Analogies Between Linearly Chirped and Colored Double Pulses in the Femtosecond Regime. Opt. Commun. 2009, 282, 227-235.

(45) Yu, Z.; Chen, X.; Weng, Y.; Zhang, J.-y. Nonlinear Chirp Effect Introduced by Kerr Medium as Optical Switches in Ultrafast TimeResolved Measurements. Opt. Lett. 2009, 34, 1117-1119.

(46) Ajdarzadeh Oskouei, A.; Tortschanoff, A.; Braem, O.; van Mourik, F.; Cannizzo, A.; Chergui, M. Three Pulse UV Photon Echo Studies of Molecules in Solution: Effect of the Chirp. J. Chem. Phys. 2010, 133, 064506/1-064506/2.
(47) Wand, A.; Kallush, S.; Shoshanim, O.; Bismuth, O.; Kosloff, R.; Ruhman, S. Chirp Effects on Impulsive Vibrational Spectroscopy: A Multimode Perspective. Phys. Chem. Chem. Phys. 2010, 12, 21492163.

(48) Zhang, H.; Zhang, S.; Lu, C.; Jia, T.; Wang, Z.; Sun, Z. SinglePhoton Fluorescence Enhancement in IR144 by Phase-Modulated Femtosecond Pulses. Chem. Phys. Lett. 2011, 503, 176-179.

(49) Berg, M. Viscoelastic Continuum Model of Nonpolar Solvation. 1. Implications for Multiple Time Scales in Liquid Dynamics. J. Phys. Chem. A 1998, 102, 17-30.

(50) Berg, M. A.; Hubble, H. W. A Viscoelastic Continuum Model of Non-Polar Solvation.: II. Vibrational Dephasing in Moderate to HighViscosity Liquids and Glasses. Chem. Phys. 1998, 233, 257-266.

(51) Berg, M. A.; Rector, K. D.; Fayer, M. D. Two-Pulse Echo Experiments in the Spectral Diffusion Regime. J. Chem. Phys. 2000, 113, 3233-3242.

(52) Passino, S. A.; Nagasawa, Y.; Fleming, G. R. Three Pulse Stimulated Photon Echo Experiments as a Probe of Polar Solvation Dynamics: Utility of Harmonic Bath Models. J. Chem. Phys. 1997, 107, 6094-6108.

(53) Coello, Y.; Lozovoy, V. V.; Gunaratne, T. C.; Xu, B. W.; Borukhovich, I.; Tseng, C. H.; Weinacht, T.; Dantus, M. Interference without an Interferometer: A Different Approach to Measuring, Compressing, and Shaping Ultrashort Laser Pulses. J. Opt. Soc. Am. B: Opt. Phys. 2008, 25, A140-A150.

(54) Pastirk, I.; Resan, B.; Fry, A.; MacKay, J.; Dantus, M. No Loss Spectral Phase Correction and Arbitrary Phase Shaping of Regeneratively Amplified Femtosecond Pulses Using MIIPS. Opt. Exp. 2006, 14, 9537-9543. 